\title{
A Probable Case of Aspiration Pneumonia Caused by Candida glabrata in a Non-neutropenic Patient with Candidemia
}

\author{
Tsutomu KobaYASHI****, Yoshitsugu MiYAZAKI*, Katsunori YANAGIHARA**, \\ Hiroshi KAKEYA*, Hideaki OHNO*, Yasuhito HigASHIYAMA*, Yoichi HIRAKATA*, \\ Yohei MizUTA*, Kazunori TOMONO****, Takayoshi TASHIRO* and Shigeru KOHNO*****
}

\begin{abstract}
Primary Candida pneumonia is rare, and detailed reports of Candida glabrata pneumonia have not been described. A 71-year-old woman had been treated for heart failure and developed aspiration pneumonia, which was refractory to antibacterial treatment. Antifungal treatment against $C$. glabrata resulted in resolution of pneumonia and candidemia. We report a probable case of $C$. glabrata pneumonia.
\end{abstract}

(Internal Medicine 44: 1191-1194, 2005)

Key words: aspiration pneumonia, Candida glablata, candidemia, dysphagia, Gram stain

\section{Introduction}

Candida glabrata is considered a relatively nonpathogenic saprophyte of the normal flora in healthy individuals, rarely causing serious infection in humans (1). However with the increasing number of immunosuppressive patients who often receive antimicrobial and antifungal treatments, the incidence of mucosal and systemic infections caused by $C$. glabrata has increased significantly $(2,3)$.

Candida pneumonia seems to exist in two forms. The most common form is a hematogenously disseminated pulmonary infection along with various other organs, often resulting in fungal emboli in the lung. The other form is rare and occurs after aspiration of oropharyngeal material, causing primary pneumonia (4).
Because Candida spp. are part of the normal flora (and hence the difficulty in judging an isolated Candida in the respiratory tract as the pathogen), a firm diagnosis of Candida pneumonia is often difficult, and confirmation generally requires histopathological examination. Although several epidemiological reports showed $C$. glabrata as a pathogen of lower respiratory infections $(5,6)$, a case report of primary $C$. glabrata pneumonia has not been described. We report a probable case of $C$. glabrata pneumonia in which the diagnosis was established by the appearance of new pulmonary infiltrates, positive blood culture, positive sputum culture and sputum smear examination.

\section{Case Report}

A 71-year-old woman was admitted to our hospital for the treatment of chronic heart failure associated with aortic valve insufficiency. The past medical history was notable for the excision of a tongue tumor three years earlier. She had been receiving nasogastric feeding and aspiration pneumonia occurred several times since glossectomy. She was treated with oral furosemide (40 mg daily), losartan ( $25 \mathrm{mg}$ ), intravenous human atrial natriuretic polypeptide (HANP, $0.05 \mu \mathrm{g} / \mathrm{ml} / \mathrm{h}$ ) and dopamine $(2 \mu \mathrm{g} / \mathrm{ml} / \mathrm{h})$ after admission, which resulted in a gradual improvement of subjective symptoms. No intravenous catheter was applied for medication or infusion. On the 3rd hospital day, the patient pulled out the stomach tube herself and aspiration was suspected to have occurred. She developed fever of $39.0^{\circ} \mathrm{C}$ on the next day. Laboratory data showed leukocyte count 5,800/ $\mu$ l (stab neutrophils $4 \%$, segmented neutrophils $80 \%$, lymphocytes $6 \%$, monocytes $8 \%$, eosinophils $1 \%$ ), platelet count $130,000 / \mu 1$, blood urea nitro-

From *the Second Department of Internal Medicine, **Department of Pharmacotherapeutics, ***Division of Molecular \& Clinical Microbiology, Department of Molecular Microbiology \& Immunology, Nagasaki University Graduate School of Biomedical Sciences, Nagasaki and ****Department of Infection Control, Osaka University Hospital, Osaka

Received for publication January 14, 2005; Accepted for publication June 18, 2005

Reprint requests should be addressed to Dr. Yoshitsugu Miyazaki, Second Department of Internal Medicine, Nagasaki University School of Medicine, 1-7-1 Sakamoto, Nagasaki 852-8501 

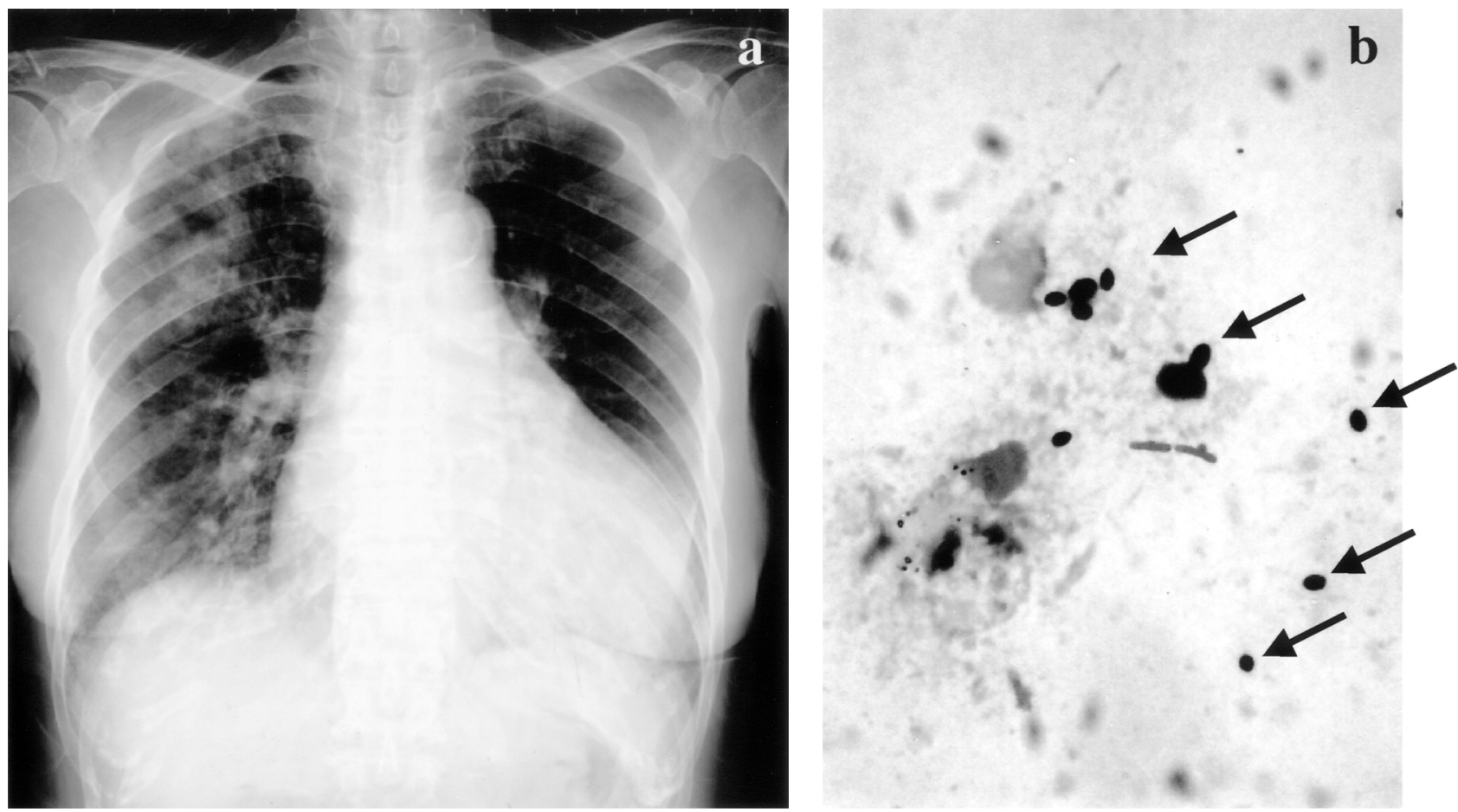

Figure 1. (a) Chest radiograph taken on 4th day of hospitalization. Infiltration shadows are seen in the right upper and lower lung fields. (b) Gram staining of sputum obtained on the same day of chest radiography. Note the presence of Gram-stained yeasts (arrows). Gram negative rods and gram positive cocci with neutrophils were seen in every view field (Magnification, $\times 1,000)$.

gen (BUN) $66 \mathrm{mg} / \mathrm{dl}$, serum creatinine $(\mathrm{Cr}) 1.4 \mathrm{mg} / \mathrm{dl}$, aspartate aminotransferase (AST) $102 \mathrm{U} / l$, alanine aminotransferase (ALT) $60 \mathrm{U} / \mathrm{l}$, total bilirubin (T-bil) $0.6 \mathrm{mg} / \mathrm{dl}$, lactate dehydrogenase (LDH) $622 \mathrm{IU} / l$, alkaline phosphatase (ALP) $385 \mathrm{IU} / l$, cholinesterase (ChE) $92 \mathrm{IU} / l$, gammaglutamyl transpeptidase (GGTP) $68 \mathrm{U} / l$, creatine kinase (CK) 2,708 IU/l, CK-MB $63 \mathrm{IU} / l$, C-reactive protein (CRP) $1.35 \mathrm{mg} / \mathrm{dl}$, and $1,3-\beta$-D-glucan $29.8 \mathrm{pg} / \mathrm{ml}$ ( $\beta$-glucan test WAKO). Chest radiograph (CXR) revealed abnormal opacities in the right upper and lower lung fields (Fig. 1a). Sputum smear showed numerous neutrophils, Gram-positive and -negative microorganisms and yeasts. She was diagnosed as aspiration pneumonia based on the underlying conditions of bed-ridden, dysphagia due to glossectomy and sputum smear findings. Antibacterial treatment with sulbactam/ampicillin (SBT/ABPC) was started at $3.0 \mathrm{~g}$ twice per day. However, the general condition did not improve; the laboratory data worsened with leukocyte count of $12,300 / \mu 1$ and CRP 14.04 $\mathrm{mg} / \mathrm{dl}$, and the chest radiographic changes in the right upper and lower lung fields worsened, irrespective of the SBT/ABPC treatment for three days.

C. glabrata was detected in blood cultures twice and was first recognized at the 6th day of hospitalization from the sample collected on the 4th hospital day. Culture of another blood sample on the 6th day also subsequently showed the growth of $C$. glabrata. Yeasts were observed in the sputum smear by Gram staining (Fig.1b). Culture of the same smearsample sputum also showed growth of $C$. glabrata, however, it was ignored because it was considered a colonized saprophyte. No significant bacteria such as anaerobes, were recovered from the sputum.

Following confirmation of candidemia, the previously cultured $C$. glabrata from the sputum was considered pathogenic, and we assumed that $C$. glabrata played a pathogenic role in aspiration pneumonia and caused candidemia. To check for possible dissemination of candidiasis in the liver, the spleen and the kidney, ultrasound examination of these organs was performed but did not detect any low echoic lesions. There was also no infectious endocarditis as confirmed by ultrasound cardiogram. Fundus examination did not reveal any lesions of endophtalmitis. Candida laryngitis was not documented by laryngoscopic examination. There was no other focus of infection apart from that of the lung. Accordingly, liposomal amphotericin B was added to SBT/ ABPC, at an initial dose of $125 \mathrm{mg}$ on the first two days, followed by $250 \mathrm{mg} /$ day thereafter. The minimum inhibitory concentrations (MICs) of amphotericin B, flucytosin (5-FC), fluconazole (FLCZ) and itraconazole were $\leq 0.5,2, \leq 0.5$, $\leq 0.5 \mu \mathrm{g} / \mathrm{ml}$ for the isolated $C$. glabrata. The introduction of antifungal treatment resulted in improvement of the clinical signs. Body temperature decreased below $37^{\circ} \mathrm{C}$ and laboratory data also improved with leukocyte count of $6,400 / \mu \mathrm{l}$, 
CRP $7.6 \mathrm{mg} / \mathrm{dl}$, and 1, 3- $\beta$-D-glucan of $13.4 \mathrm{pg} / \mathrm{ml}$. Pulmonary infiltrates both in the right upper and lower lung fields also showed improvement. C. glabrata disappeared and was never isolated since the initiation of antifungal treatment. However, T-bil increased to $4.5 \mathrm{mg} / \mathrm{dl}$ after 14 days of amphotericin B treatment combined with SBT/ABPC. This was considered an adverse effect to the antifungal or antibacterial agent. Accordingly, we replaced amphotericin B with fluconazole and discontinued SBT/ABPC. The abnormal laboratory findings of T-bil soon decreased to $1.6 \mathrm{mg} / \mathrm{dl}$. Both candidemia and aspiration pneumonia had improved and were considered cleared.

\section{Discussion}

There is little information backed by clinical research on whether antifungal treatment should be started at the time of detection of yeasts by microscopic examination or when colonization is detected though such information is not judged significant. Clinical studies of presumptive Candida treatment are important to provide guidelines for treatment. Based on the present case, there is a need to reconsider the concept of primary Candida pneumonia especially with regard to a firm diagnosis.

Candidiasis is the most common deep-seated fungal infection in clinical practice (7), however, lower respiratory tract infection, especially primary pneumonia is rare, and a firm diagnosis of Candida pneumonia is elusive but generally requires histopathological confirmation (4). The reason for the need of a strict diagnosis of Candida pneumonia is the fact that simple colonization or contamination is much more common than true pneumonia and that diagnoses based solely on microbiological data are often incorrect. In this regard, in making the diagnosis of bacterial pneumonia, the presence of respiratory symptoms, positive blood culture, positive sputum smear and culture confirm the presence of definite pneumonia. Definition of nosocomial pneumonia in a study is actually based on the appearance of a new infiltrate with one or more findings such as abscess formation, histopathology, and quantitative culture from the lower respiratory tract (8). Similarly, the criteria for pulmonary fungal infection used in a previous study did not essentially include histopathological examination (5). Although we did not obtain histopathological proof in the present case, the patient had continuously high fever for 3 days irrespective of antimicrobial treatment, symptoms suggestive of pneumonia, appearance of new non-specific infiltrates, and positive blood culture. According to the criteria of the European Organization for Research and Treatment of Cancer (EORTC) (9), this case can be a probable fungal infection based on the following factors; a host factor, two minor clinical criteria and one microbiological criterion.

Candida infection usually occurs in at risk patients. Aspiration of oropharyngeal content is considered important in Candida pneumonia and is assumed to be the mechanism of entry of the infectious microorganisms based on the observation of very high intrabronchial and intra-alveolar fungi without vascular invasion (10). Therefore, in our patient, the risk factors of aspiration such as glossectomy, and prolonged bed rest were considered important in the development of Candida pneumonia. Bacterial involvement, including anaerobes, was taken into consideration and was treated, although no significant bacteria were cultured. Neutropenia is a known important risk factor for systemic candidiasis, but not for primary Candida pneumonia. Our patient was not neutropenic, and this finding was consistent with a previous report in which only one-third of patients with primary Candida pneumonia had neutropenia (10).

The contribution of Candida spp. to nosocomial pneumonia must be considered in some clinical settings. Candida spp. are microbiologically isolated in $40 \%$ of critically-ill ventilator-assisted patients (6), and $6 \%$ of nosocomial pneumonia in infants after cardiac surgery (8). C. albicans is the most common Candida pathogen not only in systemic but also in pulmonary candidiasis $(5,6)$, and $C$. glabrata accounts for up to $20 \%$ of pulmonary Candidiasis (5). C. glabrata infections are difficult to treat and are often resistant to many azole antifungal agents including fluconazole $(2,3)$. Recent description refers to the resistance to amphotericin B use rather than fluconazole (11). Consequently, $C$. glabrata fungemia is associated with a higher mortality rate than that of $C$. albicans. In fact, Komshian et al reported a $100 \%$ mortality in 12 patients with C. glabrata fungemia (3). It could be argued, however, that the high mortality rate described by some investigators may not reflect increased virulence but the poorer state of debilitation of patients who acquire C. glabrata infection. Considering that Candida pneumonia-related hemoptysis is ominous, as described previously by Panos et al (12), patients with Candida pneumonia should be provided with more challenging treatments. Candida should be suspected in patients with nosocomial pneumonia, and such patients with Candida pneumonia should be treated with newer antifungals such as candins, and newly developed azoles such as voriconazole and intravenous itraconazole.

\section{References}

1) Haley LD. Yeasts of medical importance. Am J Clin Pathol 36: $227-$ 234, 1961.

2) Hitchcock CA, Pye GW, Troke PF, Johnson EM, Warnock DW Fluconazole resistance in Candida glabrata. Antimicrob Agents Chemother 37: 1962-1965, 1993.

3) Komshian SV, Uwaydah AK, Sobel JD, Crane LR. Fungemia caused by Candida species and Torulopsis glabrata in the hospitalized patient: frequency, characteristics, and evaluation of factors influencing outcome. Rev Infect Dis 11: 379-390, 1989.

4) Pappas PG, Rex JH, Sobel JD, et al. Infectious Diseases Society of America. Guidelines for treatment of candidiasis. Clin Infect Dis 38: 161-189, 2004.

5) Chen KY, Ko SC, Hsueh PR, Luh KT, Yang PC. Pulmonary fungal infection: emphasis on microbiological spectra, patient outcome, and prognostic factors. Chest 120: 177-184, 2001.

6) el-Ebiary M, Torres A, Fabregas N, et al. Significance of the isolation 


\section{KOBAYASHI et al}

of Candida species from respiratory samples in critically ill, nonneutropenic patients. An immediate postmortem histologic study. Am J Respir Crit Care Med 156: 583-590, 1997.

7) Edwards JE Jr. Invasive candida infections-evolution of a fungal pathogen. N Engl J Med 324: 1060-1062, 1991.

8) Tan L, Sun X, Zhu X, Zhang Z, Li J, Shu Q. Epidemiology of nosocomial pneumonia in infants after cardiac surgery. Chest 125: 410-417, 2004.

9) Ascioglu S, Rex JH, de Pauw B, et al. Invasive Fungal Infections Cooperative Group of the European Organization for Research and Treatment of Cancer; Mycoses Study Group of the National Institute of Allergy and Infectious Diseases. Defining opportunistic invasive fungal infections in immunocompromised patients with cancer and hematopoietic stem cell transplants: an international consensus. Clin Infect Dis 34: 7-14, 2002.

10) Haron E, Vartivarian S, Anaissie E, Dekmezian R, Bodey GP. Primary Candida pneumonia. Experience at a large cancer center and review of the literature. Medicine (Baltimore) 72: 137-142, 1993.

11) Nguyen MT, Weiss PJ, LaBarre RC, Miller LK, Oldfield EC, Wallace MR. Orally administered amphotericin B in the treatment of oral candidiasis in HIV-infected patients caused by azole-resistant Candida albicans. AIDS 10: 1745-1747, 1996.

12) Panos RJ, Barr LF, Walsh TJ, Silverman HJ. Factors associated with fatal hemoptysis in cancer patients. Chest 94: 1008-1013, 1988. 